International Journal of Distributed and Parallel Systems (IJDPS) Vol.4, No.1, January 2013

\title{
STUDY OF THE SENSITIVITY OF THE OFDM WIRELESS SYSTEMS TO THE CARRIER FREQUENCY OFFSET (CFO)
}

\author{
Saeed Mohseni ${ }^{1}$ and Mohammad A. Matin ${ }^{2}$ \\ ${ }^{1} \mathrm{PhD}$ Candidate in the School of Engineering and ComputerScience,University of \\ Denver, ColoradoUSA \\ saeed.mohseni@du.edu \\ ${ }^{2}$ Associate Professor in the School of Engineering and Computer Science, University of \\ Denver, Colorado USA \\ matinedu.edu
}

\begin{abstract}
As it has already been revealed, the OFDM systems are very sensitive to the CFO. CFO is one of the most important drawbacks of the OFDM wireless systems. CFO destroys the orthogonality relationship between the subcarrier and creates ICI in OFDM systems. ICI can create frequency mismatched between the transmitter and receiver; this frequency mismatch can lead to the severe performance degradation issue for OFDM wireless systems. However for having a reliable receiver the effect of the CFO must be estimated and compensated in receiver side. This paper reviews the reasons of the creating CFO and analyses its effects on the performance of the OFDM systems. The results clearly illustrate the effects of CFO.
\end{abstract}

\section{KEYWORDS}

Carrier frequency offset (CFO), CFO estimation, OFDM performance, Frequency Offset, Inter- Carrier Interference (ICI)

\section{INTRODUCTION}

About 55 years ago, Doelz et al. [1] published the idea of dividing the transmitting data into the number of interleaved bit streams and modulate numerous carriers, and then Chang [2] was the one that in the middle of the 60's, presented the basic idea of multicarrier modulation. But, it was Weinstein and Ebert [3] that brought it to the fruition; they showed how to apply the Discrete Fourier Transform (DFT) for performing the baseband modulation and demodulation. They showed how by using the DFT, we can increase and improve the efficiency of the modulation and demodulation. As we know, in order to efficiently modulate and demodulate the OFDM signals, a few pre and post tasks must be done which the most important of them are: Frequency synchronization, Carrier Frequency Offset (CFO), Peak-to-Average Power Reduction (PAPR), phase noise compensation, channel estimation, and I/Q imbalance compensation.

In OFDM systems, the term orthogonal dictates an exact mathematical relationship between the frequencies of the subcarriers. The orthogonality of the OFDM-based systems depends on the situation that transmitter and receiver work with the same frequency reference. If this doesn't happen, the orthogonality of subcarrier will be destroyed which leads to inter-carrier Interference (ICI). Strictly speaking, all of the OFDM communication systems are sensitive to the frequency synchronization in form of carrier frequency offset (CFO). CFO severely degrades the performance of the OFDM systems, therefore estimating and compensating the 
$\mathrm{CFO}$ is a must. The CFO create a $\operatorname{shift}(\delta \mathrm{f})$ in received signal, this shift can be seen in figure 1 [4].
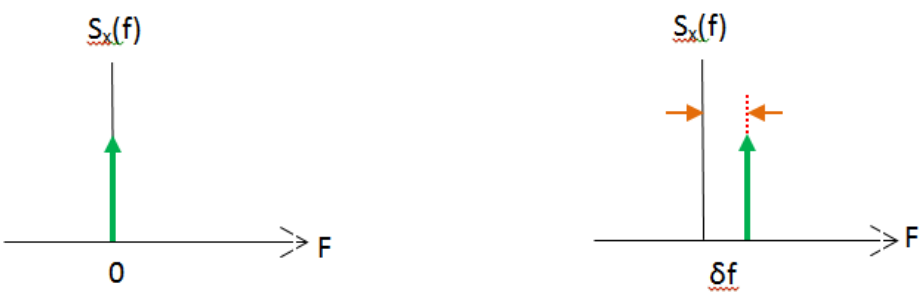

Figure1: Frequency offset $(\delta f)$

\section{CHOICE OF OFDM PARAMETERS}

The most important parameters that should be considered in any OFDM design are: guard time, symbol duration, the number of the subcarriers, subcarrier spacing, and type of the modulation. However, what dictates and assigns the selection of the parameters are the system requirements. Some of the common system requirements are: desired bit rate, available bandwidth (BW), Doppler values and acceptable delay spread. It is necessary to mention that some of these requirements can be conflicted each other. For example if we want to have a good delay spread tolerance, we should select a huge number of the subcarrier with the slight subcarrier spacing but for having a good tolerance against the phase noise the opposite is true. Therefore, there are different parameters for OFDM systems, which selecting them always leads to a tradeoff between them. Basically the three main ones that should be considered are: bit rate, bandwidth (BW), and delay spread. Since the guard time must be two to four times the Root Mean Squared of the Delay Spread (RMSDS) therefore among the mentioned parameters, the delay spread is the one that assign the guard time. Anyway, this value is related to the kind of the coding and modulation. It is worth to mention that the higher order of the QAM (i.e. 64-QAM) compare with QPSK has a higher degree of sensitivity to the ICI and ISI. The other prerequisite which has a direct impact on the selection of the parameters is the desired integer number of samples within the FFT/IFFT interval and in the symbol interval. The accepted OFDM time related and rate related parameters in IEEE 802.11 are respectively listed in table 1 and 2 [5].

\begin{tabular}{|l|l|}
\hline Parameters & Values \\
\hline Number of data sub-carriers & 48 \\
\hline Number of pilot sub-carriers & 4 \\
\hline Total number of sub-carriers & 52 \\
\hline Sub-carrier frequency spacing & $0.3125 \mathrm{MHz}$ \\
\hline IFFT/FFT period & $3.2 \mu \mathrm{s} \quad\left(1 / \Delta_{\mathrm{F}}\right)$ \\
\hline Preamble duration & $16 \mu \mathrm{s}$ \\
\hline Signal Duration BPSK-OFDM symbol & $4 \mu \mathrm{s} \quad\left(\mathrm{T}_{\mathrm{GI}}+\mathrm{T}_{\mathrm{FFT}}\right)$ \\
\hline Guard Interval (GI) duration & $0.8 \mu \mathrm{s} \quad\left(\mathrm{T}_{\mathrm{FFT}} / 4\right)$ \\
\hline Training symbol GI duration & $1.6 \mu \mathrm{s} \quad\left(\mathrm{T}_{\mathrm{FFT}} / 2\right)$ \\
\hline Symbol interval & $4 \mu \mathrm{s}\left(\mathrm{T}_{\mathrm{GI}}+\mathrm{T}_{\mathrm{FFT}}\right)$ \\
\hline Short training sequence duration & $8 \mu \mathrm{s} \quad\left(10 \mathrm{~T}_{\mathrm{FFT}} / 4\right)$ \\
\hline Long training sequence duration & $8 \mu \mathrm{s} \quad\left(\mathrm{T}_{\mathrm{GI}}+\mathrm{T}_{\mathrm{FFT}}\right)$ \\
\hline
\end{tabular}

Table 1: The timing related parameters for the OFDM systems [5] 
International Journal of Distributed and Parallel Systems (IJDPS) Vol.4, No.1, January 2013

\begin{tabular}{|c|c|c|c|c|c|c|c|}
\hline Modulation & $\begin{array}{c}\text { Coding } \\
\text { rate }(\mathrm{R})\end{array}$ & $\begin{array}{c}\text { Coded } \\
\text { bits per } \\
\text { subcarrier } \\
\left(\mathrm{N}_{\text {BPSC }}\right)\end{array}$ & $\begin{array}{c}\text { Coded } \\
\text { bits per } \\
\text { OFDM } \\
\text { symbol } \\
\left(\mathrm{N}_{\text {CBPS })}\right.\end{array}$ & $\begin{array}{c}\text { Data bits } \\
\text { per } \\
\text { OFDM } \\
\text { symbol } \\
\left(\mathrm{N}_{\text {DBPS }}\right)\end{array}$ & $\begin{array}{c}\text { Data rate } \\
(\mathrm{Mb} / \mathrm{s}) \\
(20 \mathrm{MHz} \\
\text { channel } \\
\text { spacing) }\end{array}$ & $\begin{array}{c}\text { Data rate } \\
(\mathrm{Mb} / \mathrm{s}) \\
(10 \mathrm{MHz} \\
\text { channel } \\
\text { spacing) }\end{array}$ & $\begin{array}{c}\text { Data rate } \\
(\mathrm{Mb} / \mathrm{s})(5 \\
\mathrm{MHz} \\
\text { channel } \\
\text { spacing }\end{array}$ \\
\hline BPSK & $1 / 2$ & 1 & 48 & 24 & 6 & 3 & 1.5 \\
\hline BPSK & $3 / 4$ & 1 & 48 & 36 & 9 & 4.5 & 2.25 \\
\hline QPSK & $1 / 2$ & 2 & 96 & 48 & 12 & 6 & 3 \\
\hline QPSK & $3 / 4$ & 2 & 96 & 72 & 18 & 9 & 4.5 \\
\hline 16-QAM & $1 / 2$ & 4 & 192 & 96 & 24 & 12 & 6 \\
\hline 16-QAM & $3 / 4$ & 4 & 192 & 144 & 36 & 18 & 9 \\
\hline 64-QAM & $1 / 2$ & 6 & 288 & 192 & 48 & 24 & 12 \\
\hline 64-QAM & $3 / 4$ & 6 & 288 & 216 & 54 & 27 & 13.5 \\
\hline
\end{tabular}

Table 2: The rate dependent parameters for the OFDM systems [5]

As you see in Table 2 data rate has different values, these values vary according to the type of the modulation and has a different value for BPSK, QPSK, 16QAM, and 64QAM. However the theoretical values are calculated as follows:

$$
\begin{aligned}
\frac{E_{b}}{N_{0}}=\frac{W}{R} \frac{N_{g}}{N_{S}} 10 & \frac{S N R}{10} \\
B E R & =\frac{1}{2} \operatorname{er} f_{c}\left(\sqrt{E_{b} / N_{0}}\right.
\end{aligned}
$$

Where $w$ is; signal bandwidth (BW), $N_{s}$ is symbol length, $R$ is data rate and $N_{g}$ is guard interval length.

\section{THE ORIGIN OF CFO}

Two factors can introduce the frequency offset; the first one is the lack of the matching carrier frequency between the receiver oscillator and transmitter oscillator and the second one is due to the Doppler shift. The Doppler shift can be created when there is a relative motion between the transmitter and receiver or both of them. The Doppler Effect is given as follows [4]:

$$
f_{d}=\frac{v \cdot f_{c}}{c}
$$

$f_{d}$, c and $v$ are respectively Doppler frequency, the speed of light, and the velocity of the moving receiver (i.e. $100 \mathrm{~km} / \mathrm{h}$ ). The normalized CFO $(\varepsilon)$ is:

$$
\varepsilon=\frac{f_{\text {offset }}}{\Delta \mathrm{f}}
$$

$\Delta$ fis the subcarrier spacing, the $\varepsilon$ has two portions, one integer $\left(\varepsilon_{\mathrm{i}}\right)$ and one fractional $\left(\varepsilon_{\mathrm{f}}\right)$ so:

$$
\varepsilon=\varepsilon_{\mathrm{i}}+\varepsilon_{\mathrm{f}}
$$

The instability of the oscillators is the one that can cause the mismatch between the transmitter and the receiver oscillator. The factors that can cause this instability are: temperature, humidity, the interference of the other magnetic field (which these factors are categorized as the working environment), the tolerance of the electronic elements and aging. Aging is the frequency change with time while the other factors i.e. working environment, power supply and etc. will be kept 
constant. By the way, two important factors for oscillator performance are stability and accuracy. Stability shows how well an oscillator can produce the same frequency in a specified interval of time, but the accuracy indicates the offset from the ideal value. Although the frequency errors introduce by the frequency mismatch between the local oscillator in the transmitter and thereceiver, nonetheless this frequency mismatch due to the electronics elements tolerances are avoidable. So at all the time there is a difference between the carrier frequency which has been produced in the receiver side, with the one that has been generated in the transmitted side. This frequency offset is defined as follows [4]:

$$
f_{\text {offset }}=f_{c}-f_{c}^{\prime}
$$

$\mathrm{f}_{\mathrm{c}}$ andf $\mathrm{f}_{\mathrm{c}}^{\prime}$ are respectively carrier frequency in the transmitter and the carrier frequency in receiver.Figure 2 illustrates the impact of the frequency offset. With the presence of the frequency offset, the sampling cannot be exactly done at the center frequencies of the subcarriers therefore the amplitude of the desired subcarriers will be decreased.

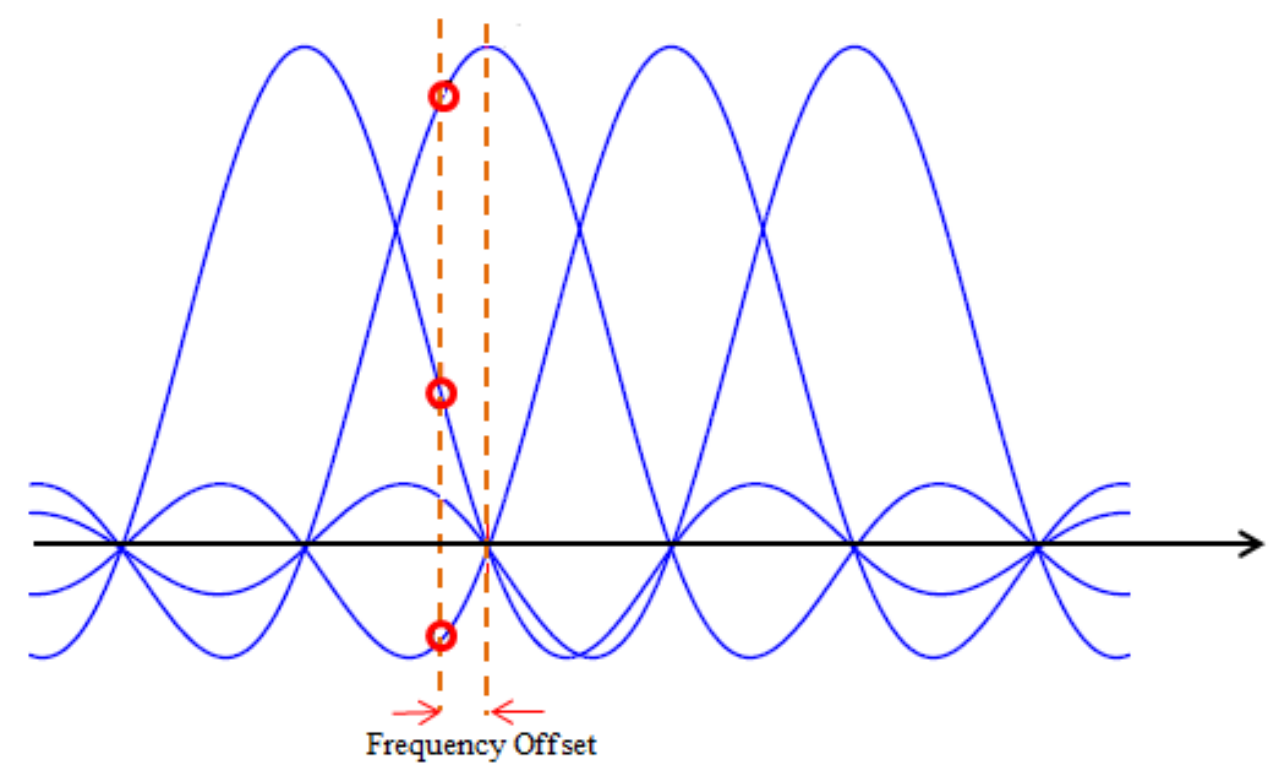

Figure2: The impact of frequency offset

\section{CFO ESTIMATION ALGORITHM AND TECHNIQUES}

Since the CFO leads to Inter-carrier Interference (ICI) therefore its degradation on OFDM systems is more than the effect of noise. However, the number of numerous proposed CFO estimation techniques and algorithm is an evidence of its importance. However these various techniques can usually be classified into two major branches:

\section{Training based algorithm}

\section{Blind algorithm and Semi-blind algorithm}

In training based algorithm, the training sequences are sent with the transmitted signal, which receiver can use them. This training sequences cause to limit the number of computation at 
International Journal of Distributed and Parallel Systems (IJDPS) Vol.4, No.1, January 2013

receiver, therefore these algorithms have a low computational complexity. In contrast, since the training sequences are needed to be sent during the signal transmission, it will lead to the reduction of the effectiveness of the data throughput.

Blind CFO estimation algorithm is the other one that is used for CFO estimation. In these methods, the statistical properties of the received signal are used for estimating the CFO. These algorithms do not need to any kind of the training sequences, thus it will not have any training overhead. on the other hand, nothing will be send with the transmitted signal that the receiver can use it for estimating CFO, therefore the receiver will not have any knowledge base of the transmitted signal, so it has to start from scratch, therefore these algorithms compare with the trained based has the higher computational complexity. Figure 3 shows the block diagram of a typical OFDM receiver with frequency synchronization.

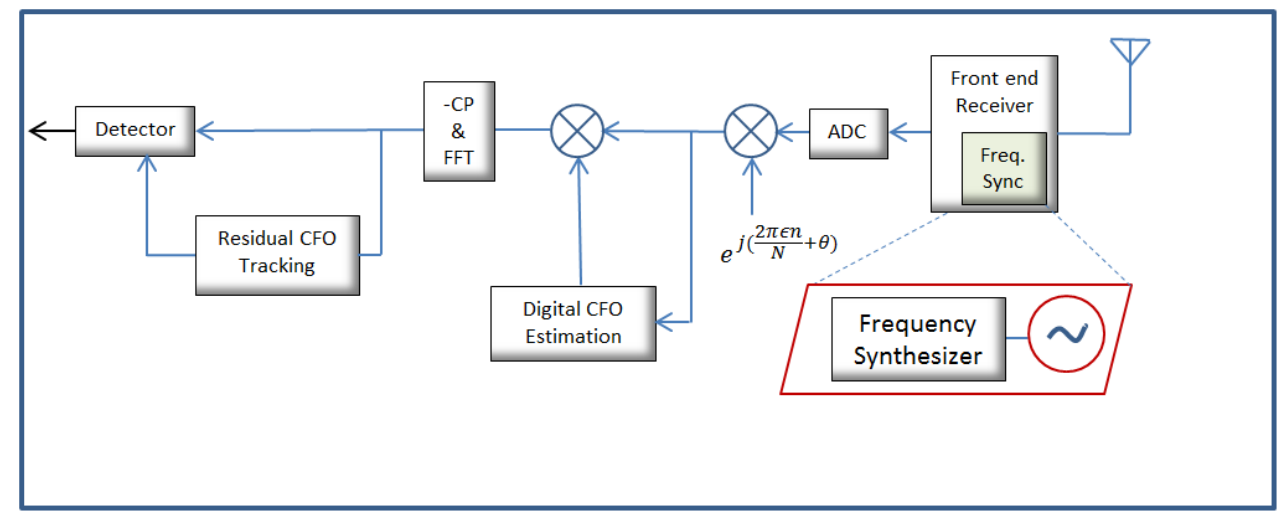

Figure 3: The block diagram of a typical OFDM receiver with frequency synchronization

\section{MODEL FOR CFO}

Figure 4 illustrates the block diagram of the OFDM wireless transceiver system.Let's consider this figure with $\mathrm{N}$ subcarriers with the sampling rate $1 / T_{S}$

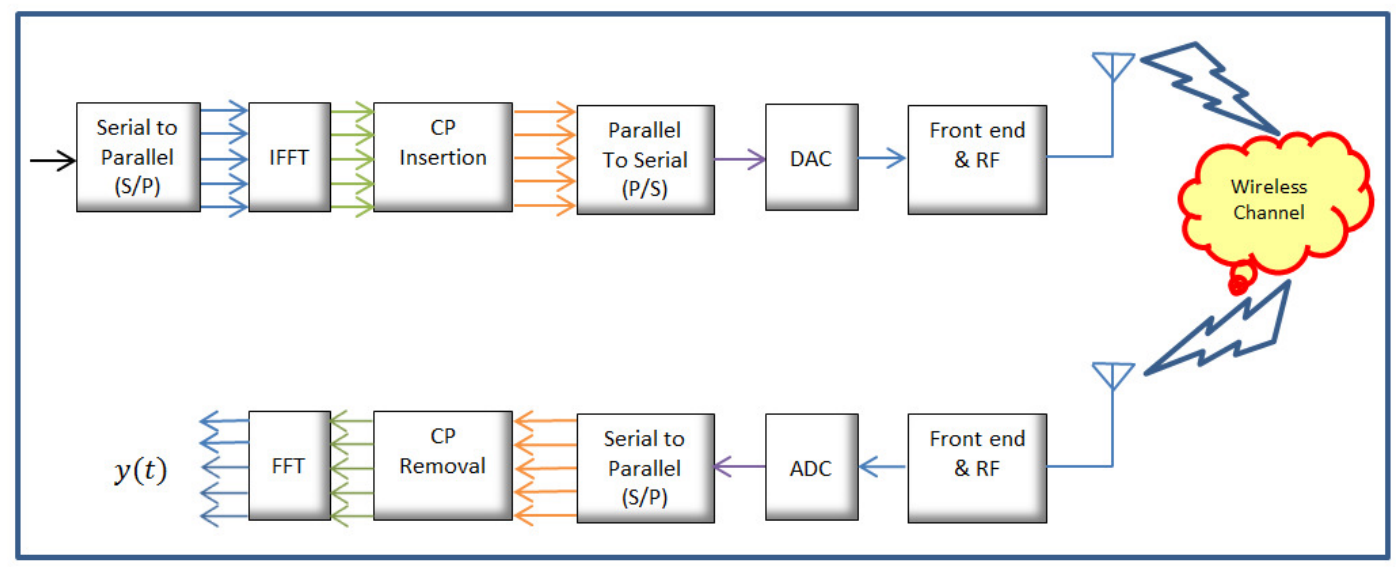

Figure 4: block diagram of the OFDM wireless transceiver system [4]

Therefore we have: 
International Journal of Distributed and Parallel Systems (IJDPS) Vol.4, No.1, January 2013

$$
\begin{aligned}
& X(n)=W_{P} S(n) \\
& y(n)=\left[y_{0}(n) y_{1}(n) y_{2}(n) \ldots y_{N-1}(n)\right]^{T} \\
& y(n)=W_{P} H s(n)
\end{aligned}
$$

In where

$$
H=\left[\begin{array}{ccc}
H(1) & \cdots & 0 \\
\vdots & \ddots & \vdots \\
0 & \cdots & H(P)
\end{array}\right]
$$

After DFT unit we have:

$$
\begin{aligned}
& W_{P}^{H} y(n)=H\left[s_{1}(n) \ldots s_{p}(n)\right]^{T} \\
& y(n)=E W_{P} H s(n) e^{j \emptyset((N+L)(n-1))} \\
& E=\operatorname{diag}\left(1, e^{j \emptyset}, \ldots, e^{j(n-1) \emptyset}\right)
\end{aligned}
$$

In where, $E$ is carrier offset matrix, $W_{p}$ is a $N \times P$ matrix, $s(n)$ is data, $H$ is channel response and $\mathrm{L}$ is length of cyclic prefix.

$$
W_{P}^{H} y(n)=W_{P}^{H} E W_{P} H s(n) e^{j \varnothing(N+L)(n-1)}
$$

Here the CFO and $\emptyset$ are needed to be estimated and applied.

\section{THE EFFECT OF CFO ON THE RECEIVED SIGNAL}

A received signal without carrier frequency offset can be considered as follows:

$$
r[n]=H[n] X(n)+\omega[n] \quad \text { where } 0 \leq n \leq N-1
$$

The equation 15 can be considered if and only if the effect of CFO is ignored. In the presence of the CFO The signal can be given as [6]:

$$
\begin{gathered}
r[n]=H[n] X(n) \frac{\sin (\pi \varepsilon)}{N \sin \left(\frac{\pi \varepsilon}{N}\right)} e^{\frac{j \pi \varepsilon(N-1)}{N}}+ \\
\sum_{m=0, m \neq n}^{N-1} H[m] X(m) \frac{\sin [\pi(m-n+\varepsilon)]}{N \sin \left[\frac{\pi(m-n+\varepsilon)]}{N}\right]} e^{\frac{j \pi(m-n+\varepsilon)(N-1)}{N}}+\omega[n]
\end{gathered}
$$

Where the normalized frequency offset $(\varepsilon)$ is:

$$
\varepsilon=\frac{f_{0}}{\Delta f}
$$

The second part of the equation 16 shows the effect of the ICI.

As it can be seen in the equation 16, the amplitude will be decreased by the factor:

$$
\frac{\sin (\pi \epsilon)}{\sin \left(\frac{\pi \epsilon}{N}\right)}
$$

and the signal experiences the phase shift equal to:

$$
\theta=e^{j \pi \varepsilon(N-1) / N}
$$


On the other hand, since the adjacent subcarriers cannot be sample at their zero crossing, therefore they cause interference.

\section{SENSITIVITY TO FREQUENCY OFFSET}

When there is the different integer number of cycles in each FFT interval the orthogonality between the subcarrier will be kept, otherwise the subcarriers will not be orthogonal. Therefore we can conclude that the frequency offset will happen when the number of cycle in FFT interval will not be an integer.

In the center of the OFDM spectrum the quantity of the Inter-Carrier Interference (ICI), in compare with their amount at the band edges, is almost double. This increase comes from the interfering of the subcarriers from both sides on the middle ones. However the degradation of the SNR which is introduced by the frequency offset can be given by [7]:

$$
D_{\text {freq }} \cong \frac{10}{3 \ln 10}(\pi \Delta f T)^{2} \frac{E_{S}}{N_{o}}
$$

Where $D_{\text {freq }}, T, E_{b}$ and $N_{0}$ are in order; frequency offset, symbol duration, energy per bit (for OFDM signal) and one sided noise Power Spectrum Density (PSD).

Figure 5 illustrates the SNR degradation as a function of the frequency offset to the subcarrier spacing. For this illustration we consider two different values for $E_{S} / N_{0}$ which are 18 and 16 $\mathrm{db}$.However the maximum acceptable frequency offset can only happen when the frequency offset is less than one percent of the subcarrier space. As a result, for overcoming to the mentioned problem the frequency synchronization must be used before the FFT.

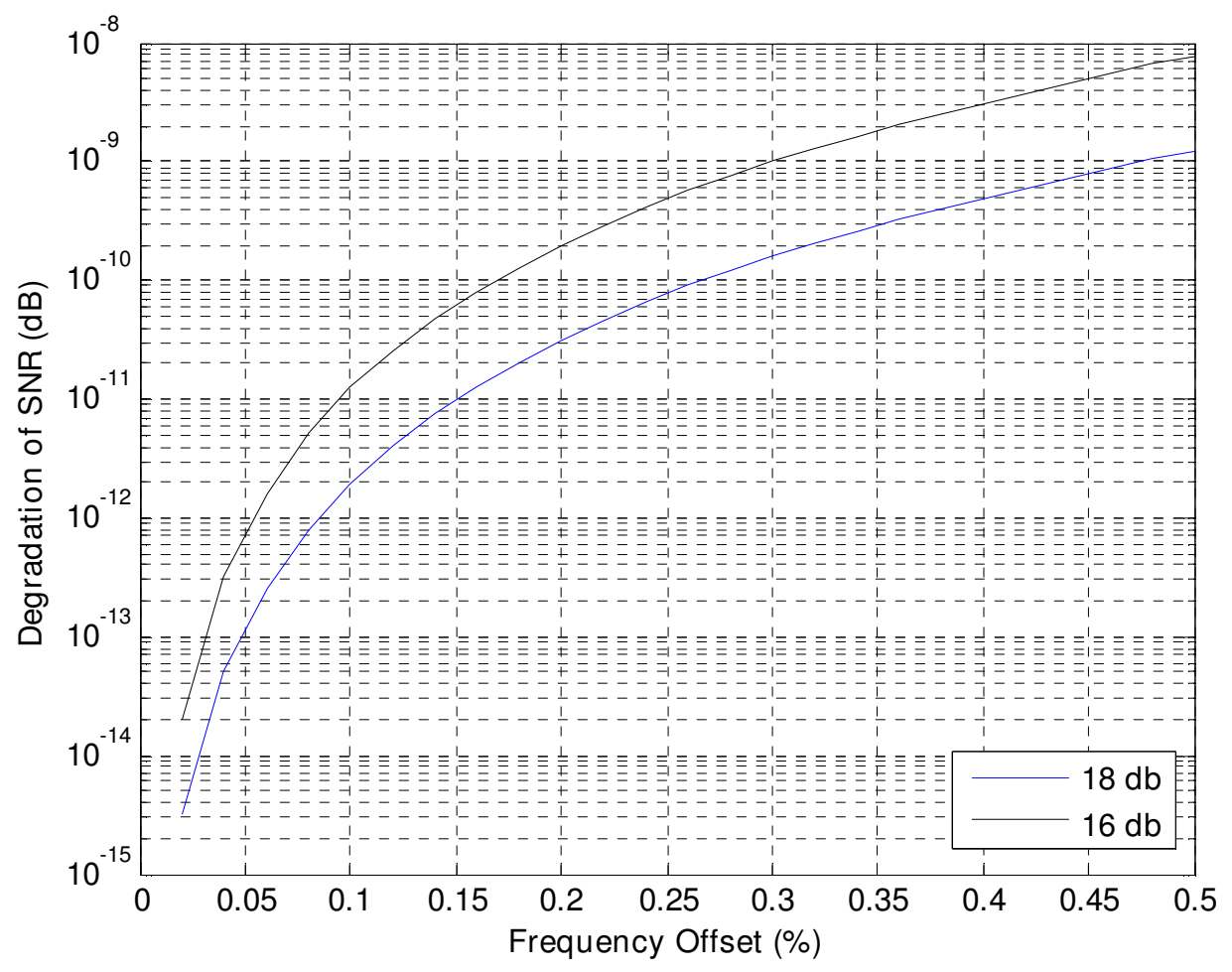

Figure 5: SNR degradation as a function of the frequency offset 
International Journal of Distributed and Parallel Systems (IJDPS) Vol.4, No.1, January 2013

\section{THE EFFECT OF INTEGER AND FRACTIONAL PART OF CFO}

As we have previously mentioned the normalized CFO has made of two parts: integer part $\left(\varepsilon_{\mathrm{i}}\right)$ and fractional part $\left(\varepsilon_{\mathrm{f}}\right)$. The integer part causes that the received signal to the receiver experiences a cyclic shift. Now let's see the effect of the fractional part. The received signal in time domain can be considered as follows [8]:

$$
Y_{l}[k]=\sum_{n=0}^{N-1} y_{l}[n] e^{-j 2 \pi k n / N}=H_{l}[k] X_{l}[k]+Z_{l}[k]
$$

$Y_{l}[k], H_{l}[k], X_{l}[k]$ and $Z_{l}[k]$ represent the received symbol, channel frequency response, transmitted symbol, and the noise in the frequency domain for the $\mathrm{k}^{\text {th }}$ subcarrier.

$$
\operatorname{IDFT}\left\{Y_{l}[k]\right\}=y_{l}[n]=\frac{1}{N} \sum_{k=0}^{N-1} H[k] X_{l}[k] e^{2 j \pi(k+\varepsilon)(n+\delta) / N}+Z_{l}[n]
$$

Where $Z_{l}[n]=I D F T\left\{Z_{l}[k]\right\}$. To see the effect of the CFO, we discard the phase noise and merely consider the $\mathrm{CFO}$ [9], therefore the equation 22 can be written as:

$$
y_{l}[n]=\frac{1}{N} \sum_{k=0}^{N-1} H[k] X_{l}[k] e^{2 j \pi(k+\varepsilon) / N}+Z_{l}[n]
$$

The FFT of the equation 23yields [8]:

$$
\begin{gathered}
y_{l}[k]=F F T\left\{y_{l}(n)\right\} \\
y_{l}[k]=\frac{\sin \pi \varepsilon_{f}}{N \sin \left(\pi \varepsilon_{f} / N\right)} e^{\left.j \pi \varepsilon_{f}(N-1)\right) / N} H_{l}[k] X_{l}[k]+I_{l}[k]+Z_{l}[k]
\end{gathered}
$$

The first part of the equation 25 shows the effect of the Fractional part of the CFO. The figures 6 and 7 show its effect. However as we expected; by increasing the fractional part of the CFO the effect of it on phase distortion and amplitude increases. This can be seen in figures 6 and 7 . In these figures the effect of noise and STO are not considered at all.

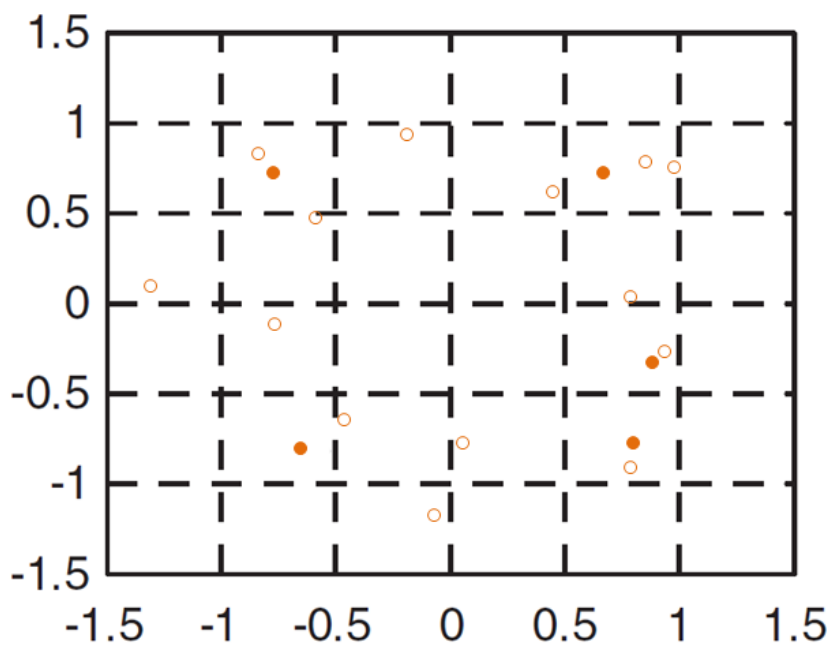

Figure 6: The effect of CFO when $\varepsilon=0.4$ 
International Journal of Distributed and Parallel Systems (IJDPS) Vol.4, No.1, January 2013

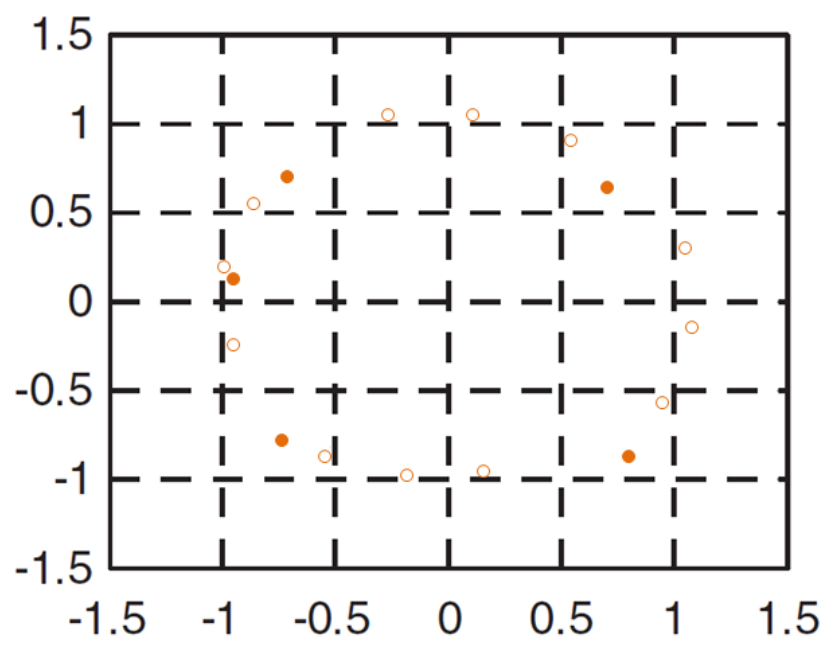

Figure 7:The effect of CFO when $\varepsilon=0.06$

\section{THE EFFECT OF CFO ON PHASE SHIFT}

The figure 8 illustrates the effect of CFO on the phase in the time domain and figure 9 presents the phase change between them. Here we consider the system is not exposing to any noise. The solid line in figure 8 shows when there is no CFO (i.e. perfect case), and dash lines shows when there is $\mathrm{CFO}$.

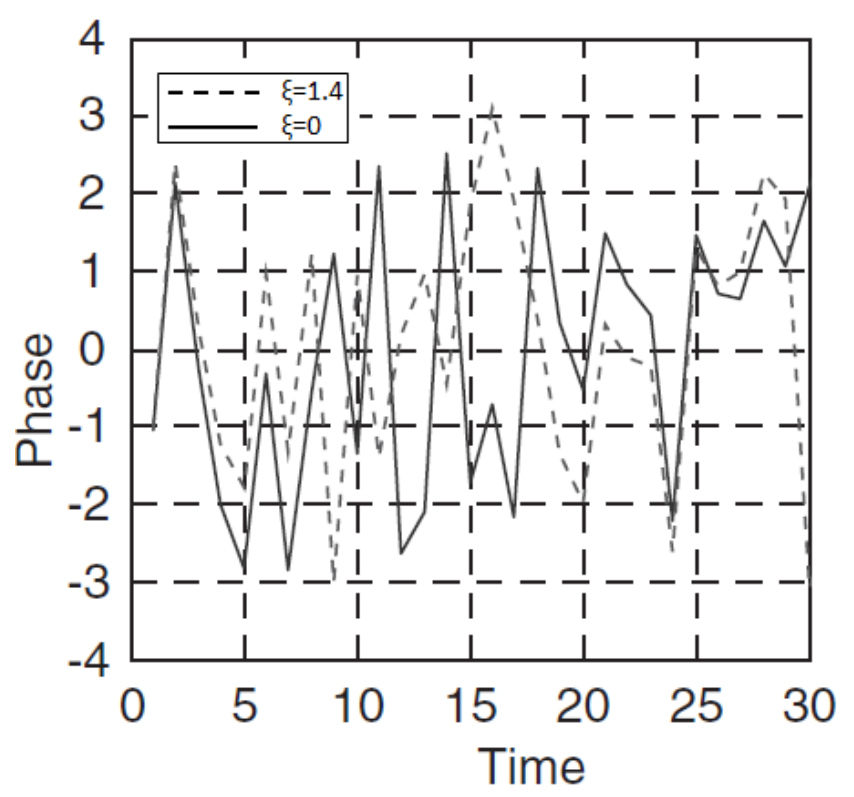

Figure 8: The effect of $\mathrm{CFO}(\xi)$ on phase in the time domain 
International Journal of Distributed and Parallel Systems (IJDPS) Vol.4, No.1, January 2013

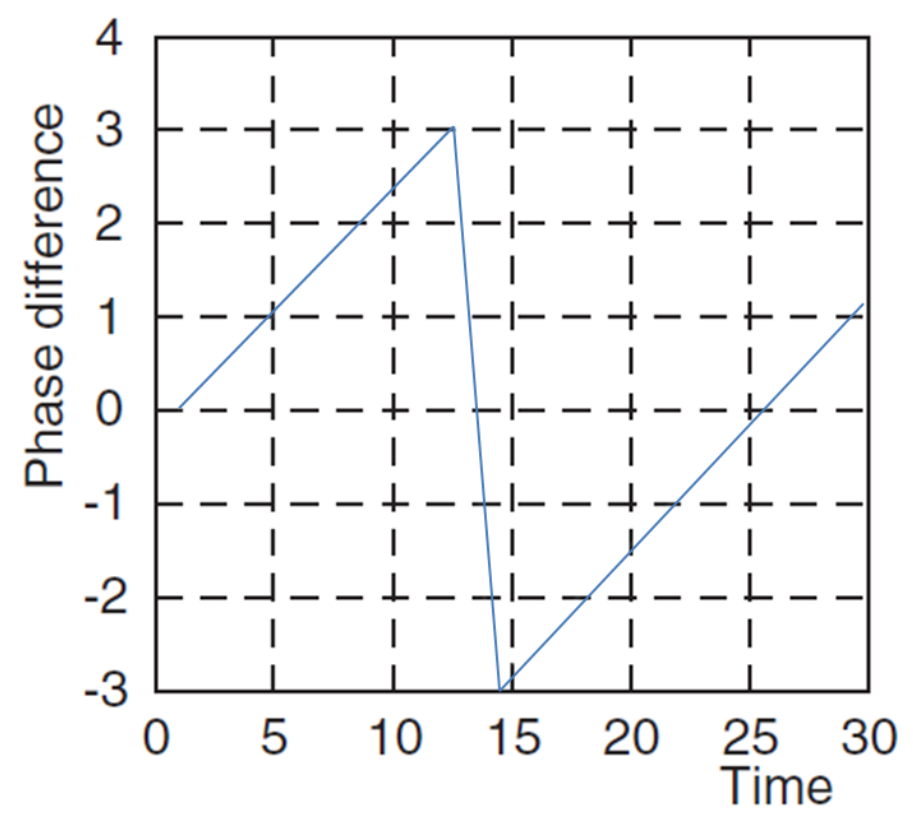

Figure 9: The effect of CFO $(\xi)$ on phase difference

\section{Estimating CFO}

The algorithms that use $\mathrm{CP}$ for estimating $\mathrm{CFO}$, can only estimate the $\mathrm{CFO}$ in a limited range. This limited range is:

$$
-\frac{1}{2} \leq \varepsilon \leq \frac{1}{2}
$$

To increase this limit, it is needed to decrease the distance between two blocks of samples for correction, which this can be done by using training symbol. To increase the CFO estimation within a bigger range we can use equation $27[10,11]$.

$$
\hat{\varepsilon}=\frac{D}{2 \pi} \arg \left\{\sum_{n=0}^{N / D-1} y_{l}^{*}[n] y_{l}[n+N / D]\right\}
$$

Where $N / D$ is an integer; Here the range is:

$$
-\frac{D}{2} \leq \varepsilon \leq \frac{D}{2}
$$

The CFO estimation range by using equation 27 will be increased to $|\varepsilon| \leq D$. However this increase cost to the degradation of the MSE performance. Figure 10 shows the effect of increasing the estimation range of $\mathrm{CFO}$ versus the MSE performance for $\mathrm{D}=1$ (red graph) and $\mathrm{D}=5$ (black graph). 


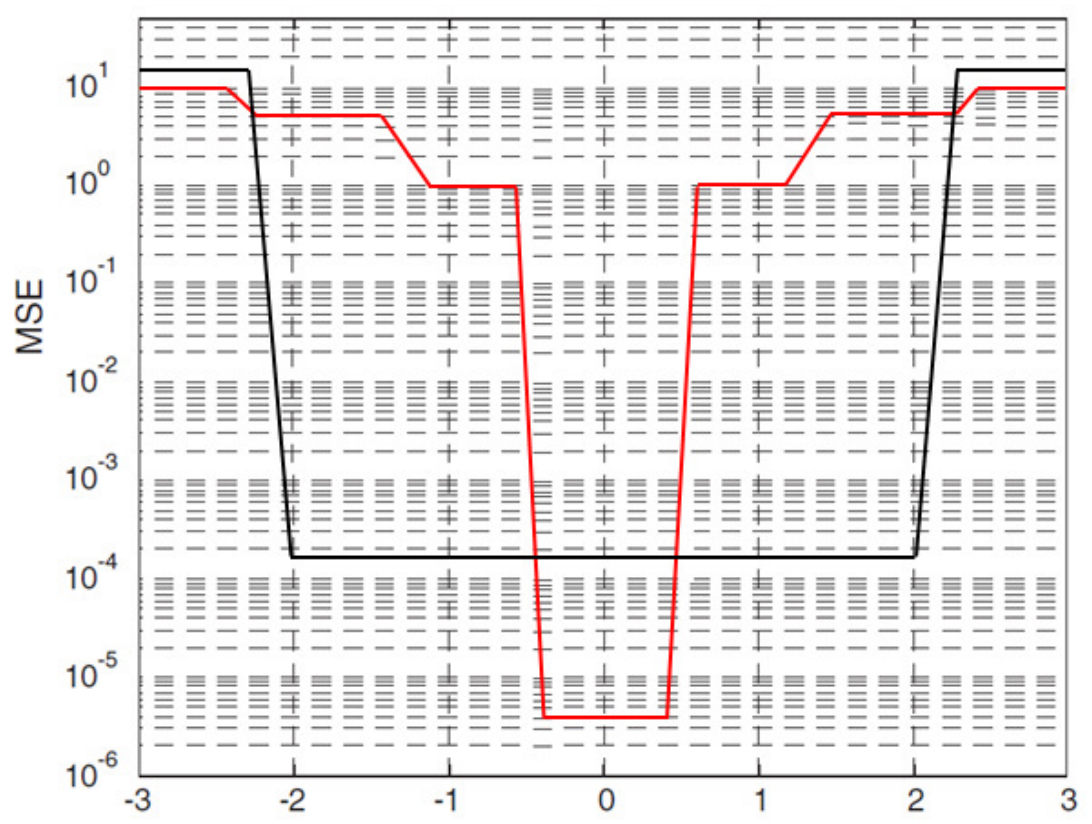

Figure10: CFO VS the MSE for D=1 (Red graph) and D=5 (Black Graph)

\section{DEgRAdATION OF OFDM SYSTEMS DUE TO THE CFO}

Thecomplex transmitted OFDM signal in a period $\mathrm{T}$ at the frequency $\mathrm{m} / \mathrm{T}$ can be state as [12]:

$$
s(t)=\left(\sum_{m=0}^{N-1} a_{m} e^{\frac{j 2 \pi m t}{T}}\right) e^{j \theta(t)}
$$

In which $a_{m}$ is data symbol and the term $\theta(t)$ represents the time varying phase due to the carrier frequency offset between transmitter and receiver.

With an accepted approximation the degradation in terms of $\mathrm{dB}$ is defined as follows [12]:

$$
D \cong \frac{10}{\operatorname{Ln} 10}\left(\left(1-E_{0}^{2}\right)+V_{0} \frac{E_{S}}{N_{0}}\right)
$$

In which, the signal to noise ratio (SNR) is: $E_{S} / N_{0}$, and $V_{0}$ is the variance for others noises and $E_{0}{ }^{2}$ is the power of the component.

In case of the presence of the frequency offset $(\Delta f)$ between the transmitter and receiver, the $\theta(t)$ in equation 29 willbe defined as follows:

$$
\theta(t)=2 \pi \Delta f t+\theta_{0}
$$

Therefore the degradation (D) for OFDM is defined as [12]: 


$$
D \cong \frac{10}{3 \operatorname{Ln} 10}\left(\pi N \frac{\Delta f}{R}\right)^{2} \frac{E_{S}}{N_{0}}
$$

From equation32, it is obvious that the degradation for an OFDM is proportional with the square root of the frequency offset and it is also proportional with $E_{s} / N_{0}$. In another word, OFDM is very sensitive to frequency offset; the frequency offset can cause degradation in OFDM systems.

\section{CONCLUSIONS}

In this paper a brief framework for carrier frequency offset (CFO) is provided. The importance of the study of the carrier frequency offset estimation and the source of the CFO for OFDM systems has been reviewed. We showed the CFO causes the received signal rotates quicker in time domain when the phase differences increase linearly. We have also showed; increasing the estimation range leads to decrease the MSE performance. However it is shown that the system performance can severely be affected by CFO. Further study can be carried out in this area by considering the other parameters which have the impact on degrading the performance of the OFDM systems.

\section{REFERENCES}

[1] Lee, Doelz, M.L.; Heald, E.T; Matin, D.L.; "Binary Data Transmission Techniques for Linear Systems" Proceeding of the IRE, Volume 45, pages 656-661; May 1957

[2] R.W. Chang; “Orthogonal Frequency Division Multiplexing”, U.S. Patent 3,488,455, filed 1960 's issued Jan, 1970

[3] Weinstein, S. B., and P. M. Ebert, "Data Transmission of Frequency Division Multiplexing Using the Discrete Frequency Transform" IEE Transaction on Communications, Volume 19, No. 5, October 1971, pp. 628-634

[4] Saeed Mohseni, Mohammad A. Matin "Study of the estimation techniques for the Carrier Frequency Offset (CFO) in OFDM systems" IJCSNS International Journal of Computer Science and Network Security, VOL.12 No.6, June 2012

[5] IEEE Standards Association; http://standards.ieee.org/getieee802/download/802.112012.pdfhttp://standards.ieee.org/about/get/802/802.11.html

[6] P. H. Moose, "A technique for orthogonal frequency division multiplexing frequency offset correction" Communications, IEEE Transaction on Oct 1994, volume 42, issue 10, pages 29082914

[7] Pollet, T., Van Bladel, M. Moeneclaey; "BER sensitivity of OFDM systems to carrier frequency offset and Wiener phase noise" Communications, IEEE Transactions on Feb/Mar/Apr 1995 pages: 191-193

[8] Muschallik, C "Influence of RF oscillator on an OFDM signal” IEEE Transaction on Aug 1995, volume 41, issue 3, pages: 592-603

[9] Minn, H., Zeng M., and Bhargava V. K., "On Timing Offset Estimation for OFDM Systems”, IEEE Transaction Communication Volume 4, No. 72000

[10] Schmidl, T.M., Cox, D.C. "Low-overhead, low-complexity [burst] synchronization for OFDM", IEEE, Communications, 1996. ICC '96, Conference Record, Converging Technologies for Tomorrow's Applications, 1996 volume 3, pages: 1301-1306 
International Journal of Distributed and Parallel Systems (IJDPS) Vol.4, No.1, January 2013

[11] Schmidl, T.M. and Cox, D.C. (1997) "Robust frequency and timing synchronization for OFDM" IEEE Communication, Transaction on Dec 1997, and volume 45, pages: 1613-1621

[12] POLLET, T., Van Blade, M., and Moeneclaey, "BER sensitivity of OFDM systems to Carrier Frequency Offset and Wiener phase noise" IEEE Transaction on Communication, VOL. 43, NO. 2/3/4, Feb/Mar/April 1995

\section{Authors}

Saeed Mohseni is a $\mathrm{PhD}$ candidate from the University of Denver. He received a bachelor's degree with honor in computer science and engineering with minor in math in 2002 from the University of Colorado at Denver (UCD) in the United State and Master of Science in Computer Science in 2004 and Master of Science in Electrical Engineering in 2006 from UCD. He is also a member of Golden Key National Honor Society. Saeed has been duly licensed and authorized to practice as a Professional Engineer in Electrical and Computer Engineering in the United State. His primary research interest is the effects of PAPR, CFO, ICI, BER and path loss in wireless OFDM systems.

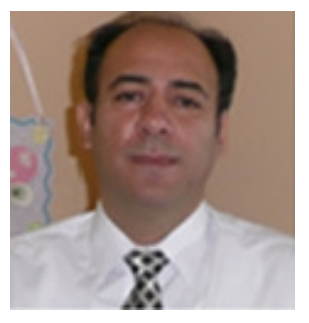

Mohammad A. Matin received a BS and an MS (Thesis) from Applied Physics and Electronics, from University of Dhaka, Bangladesh, and a PhD in Electronics and Electrical Engineering from University of Nottingham, England in 1993. After receiving a $\mathrm{PhD}$, he was a postdoctoral fellow (1994-1996) and research engineer (1996-1998) at McMaster University, Hamilton, Canada and a Senior Research Associate (1998-2000) at the University ofthe Toronto, Canada. He joined University of Denver as a Tenure Track Assistant Professor in 2000 and was promoted with tenure in 2006. Currently he is an Associate Professor of Electrical and Computer Engineering, at the School of Engineering and Computer Science, University of Denver, Colorado, USA. He is a Senior

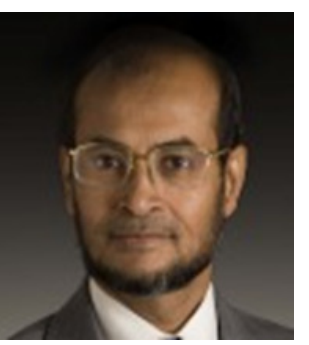
Member of IEEE, SPIE and OSA, and a member of Photonics Society, ASEE and Sigma Xi. His research interest is in Optoelectronic Devices (such as Sensors and Photovoltaic), Ultra-wide Band Radio over Fiber, Digital, Optical \& Bio-Medical Signal \& image Processing, OPGW, and Pedagogy in Engineering Education. He is the author and co-author of more than 150 journal and conference publications. 